\title{
Pluralismo y reconocimiento social de los nuevos informativos de RTVE (2006-2012)
}

\author{
Alicia GÓmEz MONTANO \\ Universidad Camilo José Cela \\ amontano@ucjc.edu
}

Recibido: $11 / 10 / 2012$

Aceptado: 23/01/2013

\begin{abstract}
Resumen
Desde el comienzo de sus emisiones en 1956, los contenidos informativos de la televisión pública estuvieron tutelados políticamente. Primero por los órganos de propaganda de la dictadura y más tarde, tras el restablecimiento de la democracia, por el partido gobernante. Durante décadas, la sociedad española asumió con cierta naturalidad, que los informativos de la radiotelevisión pública estaban al servicio del gobierno. Esta perspectiva empezó a cambiar con la reforma y la entrada en vigor de la Ley 17/2006. La principal fuente para la elaboración de este trabajo han sido los estudios realizados entre los años 2006 y 2012 por diferentes empresas especializadas, el CIS, RTVE y los debates de la Comisión de Control Parlamentario, donde queda constancia del apoyo de los ciudadanos a unos contenidos informativos independientes.
\end{abstract}

Palabras clave: Televisión pública, Control gubernamental, Percepción ciudadana, Independencia y pluralismo.

\section{Pluralism and social recognition of new newscasts of RTVE (Spain, 2006-2012)}

\begin{abstract}
Contents of the News programs of the Spanish state-owned television were politically controlled since the beginning of its existence, in 1956. Dictatorship propaganda bodies were in charge of this control at the beginning, and later, since the restoration of democracy, political party in power was monitoring those contents. Spanish society assumed without major criticism, for decades, that the news programs of the public radio and television were just for serving the government (and not the other way round). This perspective started to change with the reform of the organization and the 17/2006 Law. Main sources for the elaboration of this document have been those studies done between 2006 and 2012 by different specialized companies, CIS (Sociological Research Center) RTVE, and the debates held at the Parliamentary control commission, where the support of the citizens to the independent contents of the news services are made clear.

Keywords: Public television, Government control, Perfection of the audience, Independence, Pluralism.

\section{Referencia normalizada}

GÓMEZ MONTANO, Alicia (2013): "Pluralismo y reconocimiento social de los nuevos informativos de RTVE (2006-2012)". Estudios sobre el Mensaje Periodístico. Vol. 19, Núm. especial marzo, págs.: 233242. Madrid, Servicio de Publicaciones de la Universidad Complutense.

Sumario: 1. Introducción: planteamiento y límites cronológicos. 2. Metodología. 3. Desarrollo; 3.1. La imagen de los informativos al inicio de la reforma de RTVE. Diagnóstico interno; 3.2. Percepción del cambio tras la aprobación de la Ley $17 / 6$ de 5 de junio de 2006; 3.2.1. Desarrollo de la Ley y contexto social; 3.2.2. Estudio de IPSOS MEDIA CT (The Media Research Specialists, octubre de 2008); 3.2.3. Análisis de preferencias entre las principales cadenas de televisión; 3.3. Aceptación social de los informativos de TVE; 3.4. Balance de las intervenciones de los partidos políticos en los informativos de TVE (2004-2011); 3.5. Percepción del cambio en la Comisión Mixta de Control Parlamentario de la Corporación RTVE. 4. Conclusiones. 5. Referencias bibliográficas.
\end{abstract}




\section{Introducción: planteamiento y límites cronológicos}

El cambio de la dictadura a la democracia no se tradujo, para el audiovisual público, en pérdida de control político. Durante la transición política, "los profesionales de la televisión optaron por un equilibrio que garantizó conformidad y permisividad hacia ciertas actuaciones gubernamentales so pretexto de que únicamente se perseguía la instauración de la democracia”. (Martín Jiménez, 2012)

Aunque la ciudadanía española había asumido con cierta naturalidad la tutela política que ejercían sobre los medios públicos los partidos gobernantes, varios acontecimientos y su tratamiento en la televisión pública de ámbito estatal, provocaron reacciones de rechazo desconocidas hasta entonces. La manipulación como fenómeno social cristalizó a partir del año 2000, coincidiendo con la mayoría absoluta del Partido Popular. Tres acontecimientos actuaron, entre otros, como catalizador o detonante: el tratamiento informativo de la guerra contra Irak, a la que se oponía mayoritariamente la opinión pública española; la gestión de la crisis del petrolero y la huelga general del 20 de junio contra el llamado "decretazo", cuya cobertura provocaría, meses más tarde, la primera condena contra TVE por vulneración de derechos fundamentales.

Tras la victoria del Partido Socialista en las elecciones generales del 14 de marzo de 2004, el nuevo Presidente del Gobierno, José Luís Rodríguez Zapatero, revalidó el compromiso electoral que había adquirido de acometer la reforma de la televisión pública y renunciar a cualquier intento de presión política. Sin embargo, los pasos que se iban dando no fueron suficientes, en un primer momento, para terminar con la desconfianza que se había instalado en la sociedad española tras décadas de promesas incumplidas.

Este artículo recoge los principales datos de la comunicación que la autora presentó al Congreso Cuiciid2012, en la que se analizaba la aceptación social de los informativos de RTVE tras la reforma que se inició en 2004 y que culminó en la Ley 17/2006. El momento temporal elegido para el final del trabajo, verano de 2012, coincide con un final de etapa y con los cambios que se produjeron en la Dirección de Informativos, cuya repercusión en los contenidos, tendrá que ser evaluada en otros trabajos.

\section{Metodología}

Para la elaboración del trabajo se analizaron los datos de los barómetros, encuestas y estudios que, desde la entrada en vigor de la Ley 17/2006, han recogido la percepción ciudadana respecto a la independencia de los contenidos informativos de la televisión pública y a su aceptación social.

Su procedencia es diversa: están los realizados por la Dirección de Investigación de Contenidos de TVE, los procedentes de empresas de medición de datos como Ipsos Media, TNS-Grupo Kantar o el Centro de Investigaciones Sociológicas y asimismo se han consultado algunos de los trabajos más importantes de los investigadores especializados en el audiovisual público.

También se recogen las actas de la Comisión Mixta de Control Parlamentario, encargada de fiscalizar a la Corporación. Asimismo, se trataba de poner en contexto los datos obtenidos y el momento social y político en el que fueron recabados, así como 
de analizar los factores que han favorecido que la televisión pública liderase, en materia de pluralismo y aceptación social, las preferencias ciudadanas.

\section{Desarrollo}

\subsection{La imagen de los informativos al inicio de la reforma de RTVE. Diagnóstico interno}

En mayo de 2005, la Dirección de Contenidos de TVE realizó un informe de uso interno, con el fin de conocer cómo se percibían, en ese momento, los nuevos informativos. La Dirección de Seguimiento, lleva más de 20 años funcionando con metodología científica de trabajo. Para la elaboración del informe se entrevistó a 18 grupos de discusión, pertenecientes a todas las clases sociales y residentes de 6 comunidades autónomas con canales propios: Andalucía, Cataluña, Galicia, Madrid, País Valenciano y País Vasco. El estudio se realizó en dos fases (noviembre 2004 y febrero de 2005) para poder recoger cierta consolidación del cambio.

Una de las conclusiones fue la evidente y progresiva pérdida de poder referencial de los informativos de TVE, como consecuencia del efecto negativo arrastrado de la etapa anterior (gobierno PP y Urdaci).

"Los datos producidos en esta investigación indican que la valoración de los programas informativos de TVE tras haber alcanzado su punto más bajo con el tratamiento dado a los atentados del 11-M, estarían en pleno proceso de recuperación a raíz del cambio surgido con el nuevo gobierno estatal y la posterior sustitución del equipo que dirigía los informativos" (Dirección de InVEstigación de Contenidos De TVE, 2005)

\subsection{Percepción del cambio tras la aprobación de la Ley $17 / 6$ de 5 de junio de 2006.}

\subsubsection{Desarrollo de la Ley y contexto social}

La reforma de la radiotelevisión pública, -que recogía parte de las recomendaciones del Informe para la Reforma de los medios públicos-, permitió romper la tutela política que había durado medio siglo. La ley incluía la elección por mayoría cualificada, del Consejo de Administración y por primera vez, el Presidente de la Corporación no era designado por el Gobierno. Los mandatos pasaron a ser de 6 años, para evitar coincidencias con las legislaturas y recogía viejas aspiraciones: Derecho de acceso, Consejos de Informativos y Defensor del Espectador.

En ese momento, ni la cadena ni los informativos eran líderes de audiencia y aunque la audiencia no está necesariamente vinculada a la credibilidad, siempre es un objetivo para los gestores y profesionales del medio público. La recuperación de la audiencia de los informativos fue relativamente rápida, confirmando la tendencia que se inició en la etapa transitoria al frente de la cual estuvo Carmen Caffarel.

En marzo de 2007, los informativos consolidan el liderazgo y así continuarán, -aunque se inicia un descenso que coincide con los cambios en la Dirección de Informativos-, hasta octubre de 2012, últimos datos conocidos al redactar este trabajo. La audiencia general de la cadena tardaría más, -hasta principios del 2009-, en alcanzar el liderazgo. Al inicio de la Corporación, La 1 ocupaba el tercer lugar tras Antena 3 y Tele 5 . 


\subsubsection{Estudio de IPSOS MEDIA CT (The Media Research Specialists, octubre de 2008)}

En el año 2008 la mejora de la imagen de los nuevos informativos de TVE es un hecho y lo reflejan todos los sondeos, entre ellos el que encarga la Corporación a la empresa IPSOS y que se lleva a cabo a partir de 800 entrevistas en todo el territorio nacional, entre los días 15 y 31 de octubre de 2008.

TVE1 obtiene la puntuación más alta cuando preguntan a los entrevistados qué cadena es la más veraz y créble; imparcial y neutral; la que ofrece distintos puntos de vista, trata temas sociales o cubre bien la información política nacional. La nota media que obtiene TVE1 es del 7,2\%. Esta media ponderada sobre 10 se obtiene con las valoraciones que hacen los encuestados de los siguientes atributos: veracidad y credibilidad; imparcialidad y neutralidad; información completa y en profundidad; informa bien sobre Comunidades Autónomas; cubre bien la información política nacional; trata temas sociales; información desde distintos puntos de vista; buenos corresponsales en extranjero; cubre bien la información internacional; presta atención a la información cultural; presentadores profesionales de prestigio; pronóstico fiable del tiempo y buen información deportiva.

\subsubsection{Análisis de preferencias entre las principales cadenas de televisión}

A partir del año 2009, RTVE encarga anualmente al grupo TNS Kantar un informe de resultados sobre las preferencias del espectador. Se trata de conocer la valoración ciudadana sobre los programas informativos, debates, entrevistas etcétera. El tamaño de la muestra es significativo: 1000 entrevistas telefónicas, dentro del territorio nacional, con un error estadístico máximo de $+3,2 \%$.

El informe del año 2009, elaborado a partir del trabajo de campo que se hizo en el mes de septiembre, arroja datos positivos para la cadena y especialmente para los servicios informativos. Los informativos de La 1 también son los que ofrecen información más completa y rigurosa $(38,8 \%)$; los que cuentan con mejores profesionales $(32,1 \%)$ y los que ofrecen más puntos de vista $(25,8 \%)$. La 1 también se sitúa en la primera posición por ser el canal con mejores programas de debate, como "Tengo una pregunta para usted" y "59 segundos". El estudio concluye que en esos momentos, "La 1 es el canal preferido para mayor número de espectadores de la televisión, hecho que confirma los buenos resultados de audiencia que obtiene en los últimos tiempos"1.

Los datos son aún mejores en los años 2010 y 2011. En el estudio que realiza TNSGRUPO KANTAR para TVE en el año 2011 (fechas del trabajo de campo: del 27 de junio al 4 de julio de 2011, mediante 1.000 entrevistas en todo el territorio nacional y un error de muestra de $+-3,5 \%$ ), la diferencia entre los informativos de La 1 y sus competidores se agranda. Si en el 2009 era un 32\% de los entrevistados los que preferían informarse a través de La 1 , dos años más tarde el porcentaje ha subido hasta el $42 \%$. El $41 \%$ de los entrevistados creen que el canal que da cabida a representantes de todas opciones políticas y ofrece diversidad de puntos de vista, es La 1. Dos años antes, la cifra era del $26 \%$.

1 “Análisis de las preferencias entre las principales cadenas de televisión. Informe de resultados”. TNS. Año 2009. Página 35 
Entre los aspectos que destaca el estudio, están los arquetipos de profesionalidad e imparcialidad atribuibles a programas como Informe Semanal, 59 segundos y Los desayunos. $^{2}$

El Informe Anual de Informativos que elabora el Departamento de Marketing y Seguimiento de TVE confirma la mejora de los datos de 2011 respecto al periodo anterior. "Liderazgo informativo absoluto en todo 2011 de los Telediarios en todas y cada una de sus ediciones por cuarto año consecutivo".

Otras encuestas avalan la misma tendencia. En mayo de 2009, el diario Público da a conocer los datos del llamado Publiscopio, un estudio de preferencias a partir de los datos de 1.602 entrevistas telefónicas. Los entrevistados se decantan por los informativos de La1, incluidos los votantes del Partido Popular.

El trabajo Las televisiones y el sesgo político en la opinión pública, publicado en la colección ZOOMPolítico también es concluyente al afirmar que las audiencias más neutrales son las que se informan en TVE.

Por último, el Informe Anual sobre el estado de la profesión de la Asociación de la Prensa de Madrid (APM) correspondiente al año 2011, también concluye que los españoles "tienen una opinión claramente favorable a la labor que realiza TVE por la veracidad, la precisión y la fiabilidad de sus noticiarios". Para la elaboración de este Informe se entrevistan a 1.200 hogares de toda la geografía nacional.

\subsection{Aceptación social de los informativos de TVE}

El reconocimiento social que han tenido los contenidos informativos desde la reforma de RTVE, se debe a la percepción de pluralidad, entendida como algo más que el pluralismo político. El incremento del tiempo dedicado en los informativos de TVE a los asuntos sociales, la atención a voces críticas y la visibilidad de grupos y formaciones sin representación parlamentaria, contribuyeron a reforzar la imagen de que la televisión pública pertenece a sus ciudadanos.

Entre 2006 y 2007, un grupo de investigadores encabezados por María Lamuedra, realizaron una muestra de 40 entrevistas con profesionales de Televisión Española y la $\mathrm{BBC}$ en el marco de un proyecto de investigación. Los autores extrajeron varias conclusiones sobre hacia dónde debería dirigirse la radiotelevisión pública, que en ese momento estaba en plena transición, para consolidar su independencia y credibilidad. Un desafío que pasaba por mirar más a la sociedad y menos a la clase política.

El pluralismo social, entendido como mayor presencia de la sociedad civil en los contenidos informativos, ha ido ampliando su espacio en la radiotelevisión pública. Las noticias y reportajes con el ciudadano como principal protagonista, superaban a finales de junio del 2012, el 40\% del total de las intervenciones emitidas en los telediarios. En mayo de 2010, el Centro de Investigaciones Sociológicas dio a conocer los resultados de un barómetro sobre la imagen que los ciudadanos españoles tenían de la televisión, que confirmó el elevado grado de aceptación que había, en ese momento, hacia los contenidos de la televisión pública. Se hizo con los datos de 2.500 entrevistas a domicilio, a ciudadanos de 47 provincias.

2 "Preferencia en la elección de los canales de televisión. Imagen y posicionamiento de TVE". Encuesta de TNS-Grupo Kantar. 2011. Página 43. 
Gráfico 1. Porcentaje presencia sociedad civil en informativos diarios

\section{TODAS LAS INTERVENCIONES}

ENERO - JUNIO 2012

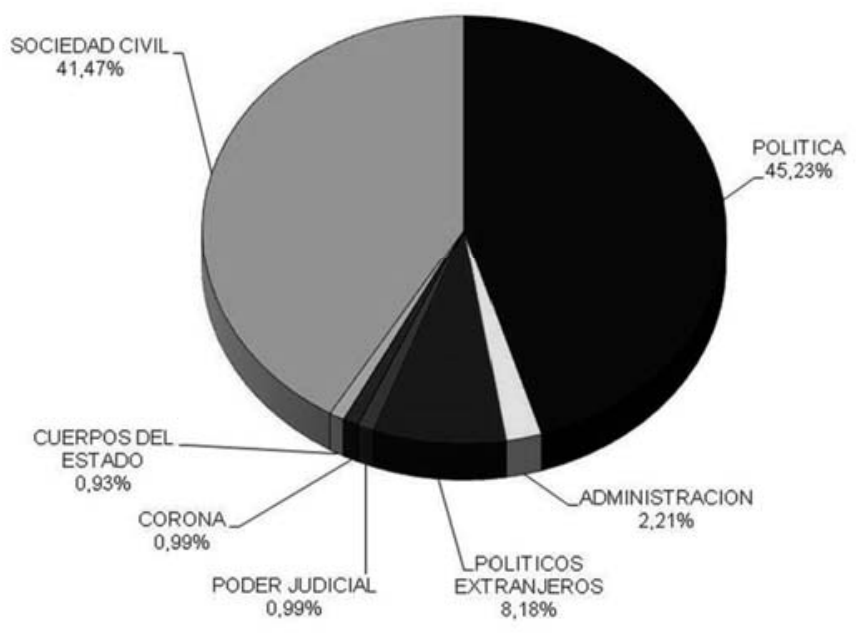

Fuente: Análisis de contenidos y seguimiento de PP.II de TVE

La 1 era el canal favorito para el 23,7 por ciento de los entrevistados, a mucha distancia de la segunda opción (Antena 3 con un 14,9\%). El estudio del CIS confirmaba la imbatibilidad de La 1 en cuanto a preferencias ciudadanas a la hora de informarse.

\subsection{Balance de las intervenciones de los partidos políticos en los informativos de TVE (2004-2011)}

En octubre de 2011, en vísperas de las elecciones que darían la mayoría absoluta al Partido Popular, el Departamento de Seguimiento de TVE elaboró un informe interno en el que recopilaba las intervenciones de los partidos políticos en los informativos de la pública desde el año 2004. Cuando la Dirección de informativos tomó posesión en el año 2004, optó por conservar tanto al equipo humano como la metodología de seguimiento por lo que los datos desde el 2004 y hasta finales de 2011 son homologables. Gracias a ello se ha podido mantener un patrón común de comparación año a año sin alteración y obtener series históricas que permiten comparar los telediarios desde hace más de dos décadas.

El Informe confirma que a finales del año 2011 se mantiene la tendencia iniciada en el 2004 de incremento de la presencia del principal partido de la oposición $(+7,6 \%)$ y de la oposición en su conjunto $(+10,3 \%)$. Asimismo, la suma de los tiempos asignados al Gobierno+el partido que le apoya, se mantiene dentro del estándar habitual en el resto de países europeos respecto a los grupos de oposición.

La lectura de los datos de presencia de los políticos en los informativos de TVE se hace partiendo de dos premisas básicas: el protagonismo informativo del Gobierno, y 
el estudio de las tendencias a largo plazo, ya que la agenda de los informativos viene fijada por la actualidad y analizarlos en un periodo de tiempo demasiado corto (inferior a dos meses) puede dar lugar a conclusiones erróneas.

"Un telediario no es un parlamento, -concluye-. A lo largo de estos últimos años hemos demostrado que podemos cumplir con ambos requisitos a la vez. Es posible elegir aquellas noticias más destacadas, facilitando que las distintas voces que tienen algo que decir en el debate público estén presentes en nuestros informativos" (TVE MARKETING, 2011)

El Informe reconoce que el pluralismo es mucho más que la presencia de los políticos.

Gráfico 2. Intervenciones de líderes de partidos políticos. De enero a diciembre de 2003 (Gobierno PP)

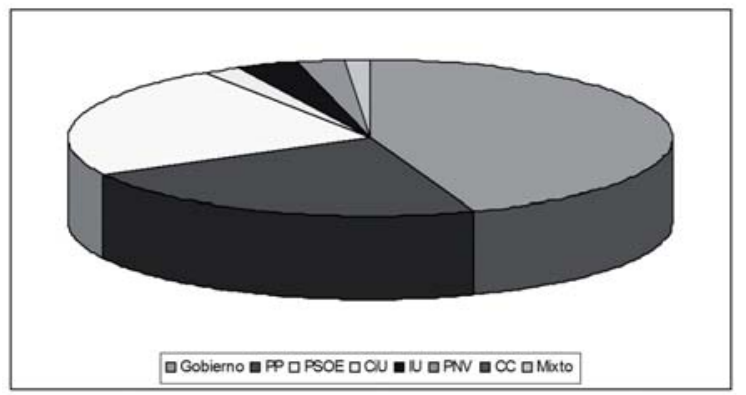

\begin{tabular}{|l|r|r|}
\hline Intervenciones & \multicolumn{1}{|c|}{ Tiempo } & \multicolumn{1}{|c|}{$\%$} \\
\hline Gobierno & 27h 07m 19s & 44,43 \\
\hline PP & 13h 50m 07s & 22,67 \\
\hline PSOE & 14h 32m 35s & 23,83 \\
\hline CiU & $1 \mathrm{~h} \mathrm{07m} \mathrm{17s}$ & 1,84 \\
\hline IU & 2h 01m 00s & 3,3 \\
\hline PNV & 1h 30m 53s & 2,48 \\
\hline CC & $4 \mathrm{~m} \mathrm{05s}$ & 0,11 \\
\hline Mixto & $48 \mathrm{~m} \mathrm{55} \mathrm{s}$ & 1,34 \\
\hline Total & 61h 02m 11s & $\mathbf{1 0 0}$ \\
\hline
\end{tabular}

Fuente: Análisis de contenidos y seguimiento de PP.II de TVE

Gráfico 3. Intervenciones de líderes de partidos políticos. De mayo a diciembre de 2004 (Gobierno PSOE)

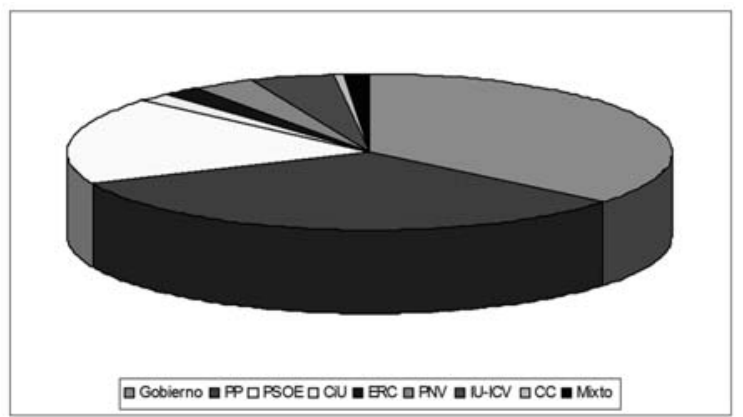

\begin{tabular}{|c|c|c|}
\hline Intervenciones & \begin{tabular}{|l|} 
Tiempo \\
\end{tabular} & $\%$ \\
\hline Gobierno & $10 \mathrm{~h} 38 \mathrm{~m} 33 \mathrm{~s}$ & 36,05 \\
\hline PP & $9 \mathrm{~h} 31 \mathrm{~m} 10 \mathrm{~s}$ & 32,24 \\
\hline PSOE & $5 \mathrm{~h} 21 \mathrm{~m} 10 \mathrm{~s}$ & 18,12 \\
\hline CiU & $34 \mathrm{~m} \mathrm{56s}$ & 1,97 \\
\hline ERC & $33 \mathrm{~m} \mathrm{19s}$ & 1,88 \\
\hline PNV & $58 \mathrm{~m} \mathrm{03s}$ & 3,28 \\
\hline IU-ICV & $1 \mathrm{~h} \mathrm{20m} \mathrm{30s}$ & 4,54 \\
\hline CC & $8 \mathrm{~m} 47 \mathrm{~s}$ & 0,5 \\
\hline Mixto & $25 \mathrm{~m} \mathrm{10s}$ & 1,42 \\
\hline Total & $29 \mathrm{~h} 31 \mathrm{~m} \mathrm{28s}$ & 100 \\
\hline
\end{tabular}

Fuente: Análisis de contenidos y seguimiento de PP.II de TVE

\subsection{Percepción del cambio en la Comisión Mixta de Control Parlamentario de la Corporación RTVE}

La percepción del cambio también tiene su reflejo en la Comisión Mixta de Control Parlamentario ante la que comparecen los Presidentes de la Corporación. Sin embargo hay que destacar que mientras las preguntas sobre pluralismo político, cultural y linguístico parten de todas las formaciones políticas, las que sugieren o afirman que los 
informativos de TVE no ofrecen una información neutral y que se manipula, proceden en todos los casos, -si exceptuamos 2 de UPyD-, del Partido Popular. Pero con la excepción de esta formación política, los partidos se interesan por el pluralismo en un sentido más cercano a los códigos de objetividad que utilizan los profesionales de la información. Y aunque la objetividad sea difícil de lograr en el discurso periodístico, en términos generales las fuerzas políticas parlamentarias reconocen que el criterio de selección pertenece a RTVE.

Del análisis de las Comisiones de Control se puede concluir que las formaciones parlamentarias están más preocupadas por el pluralismo político y por el reparto de tiempos, en función de la representación obtenida, que por el pluralismo social. Son los partidos nacionalistas, (CiU, ERC, PNV, BNG...) quienes incluyen alguna pregunta relacionada con el pluralismo lingüístico y reclaman programaciones específicas en lenguas autóctonas que reflejen la pluralidad territorial.

Durante los 5 años que transcurren desde que se aprueba en el Parlamento la ley que regula la Corporación hasta su reforma parcial, en abril de 2012, mediante un Real Decreto que modifica el sistema de elección por mayoría reforzada del Presidente y de los órganos de administración, la Presidencia de la Corporación respondió a 250 preguntas relacionadas con el pluralismo de los contenidos informativos. De ese total, 154 se referían a posibles casos de manipulación informativa y todas, a excepción de 2 de UPyD procedían del Partido Popular.

A diferencia de etapas anteriores, ya no es el conjunto de la oposición la que se queja de falta de pluralismo y contenidos gubernamentalizados. Las formaciones políticas parecen estar conformes, en términos generales, con los contenidos informativos de RTVE y sus quejas tienen que ver más con el trato exiguo a sus siglas o con la escasa presencia en debates y programas, pero no con denuncias por manipulación informativa.

Sin embargo y aún dando por sentado que RTVE alcanza en esta etapa altas cotas de independencia, es evidente que los contenidos informativos continúan parlamentarizados y sometidos a controles políticos alejados de criterios profesionales. Este escenario, -contenidos sin control gubernamental, pero fiscalizados por el Parlamento, tiene sus efectos, ya que los partidos políticos no renuncian a influir y además, consideran legítimo este objetivo (Gómez Montano, 2012).

Un Pacto de Estado en esta materia contribuiría, "al igual que otros acuerdos de primera magnitud que demandan los ciudadanos, a dar estabilidad al país y a erradicar tentaciones de intereses partidistas u otros motivos menos confesables" (Fernández Vicente, 2009)

\section{Conclusiones}

La aceptación social de los contenidos informativos de la televisión pública de ámbito estatal está vinculada más que a su reforma, al pluralismo político y social experimentado en el periodo.

Un factor decisivo ha sido el incremento del pluralismo político y del tiempo dedicado a los partidos de oposición.

La aparición de nuevos formatos, como el programa Tengo una pregunta para usted, debates como "59 segundos", y hechos puntuales como que se entrevistase 
antes a Mariano Rajoy que al Presidente del Gobierno José Luís Rodríguez Zapatero, tras ganar las elecciones en el año 2004, fueron factores que contribuyeron a visibilizar el cambio.

Más allá del pluralismo político, ha sido la pluralidad social -la presencia de voces críticas, minorías y representantes de la sociedad civil- la que ha contribuido a cambiar y a mejorar la percepción de los ciudadanos sobre un medio que consideraban gubernamentalizado.

En cuanto a la fiscalización que ejerce por Ley la Comisión de Control Parlamentario, hay que destacar que la mayoría de las formaciones políticas reconocen la aceptación de los nuevos informativos, aunque sus preguntas tienen que ver casi siempre con el reparto de tiempos a las formaciones políticas y con su presencia en los diferentes programas informativos. Este comportamiento de los partidos políticos quizás se deba al hecho de que sin son ellos lo que redactan las leyes audiovisuales y si de ellos depende su financiación y el control democrático, difícil es pedirles que se mantengan al margen.

Aunque los índices de audiencia no están necesariamente asociados ni a la credibilidad ni a la calidad del producto informativo, si se puede concluir que los índices de aceptación por parte de una amplia mayoría social, han contribuido a su liderazgo.

\section{Referencias bibliográficas}

DIRECCIÓN DE INVESTIGACIÓN DE CONTENIDOS DE TVE (2005): La imagen de los programas informativos de TVE. Madrid, RTVE.

FARIAS, Pedro (dir., 2011): Informe anual sobre la profesión periodística 2011. Madrid, Asociación de la Prensa de Madrid (APM).

FERNÁNDEZ VICENTE, Pedro (2009): "La necesidad de pactos de Estado en la política española”. Revista de Comunicación SEECI n 20, noviembre 2009. Madrid. Universidad Complutense.

GÓMEZ MONTANO, Alicia (2007): La manipulación en televisión. Madrid, Editorial Espejo de Tinta.

GÓMEZ MONTANO, Alicia (2012): "Percepción de la independencia informativa a través de los debates de la Comisión de Control de RTVE". Comunicación presentada en el Congreso Crisis y políticas. La radiotelevisión pública en el punto de mira. Universidad del País Vasco (4 y 5 de octubre de 2012)

IPSOS MEDIA CT (THE MEDIA RESEARCH SPECIALISTS) (ola octubre 2008): Análisis de resultados.

LEY 17/2006 DE 5 DE JUNIO DE LA RADIO Y LA TELEVISIÓN DE TITULARIDAD ESTATAL.

MARTÍN JIMÉNEZ, Virginia (2012): “Una aproximación a un nuevo campo de estudio sobre la transición democrática. Los inicios de la comunicación política televisiva en España". Revista de Comunicación Vivat Academia, n ${ }^{\circ} 17 \mathrm{E}$. Febrero 2012. Madrid. Universidad Complutense. 
PENADÉS, Alberto y URQUIZU, Ignacio (2011): Las televisiones y el sesgo politico en la opinión pública. Número 2011/06. Colección ZOOMPolítico. En www.falternativas.org

PUBLISCOPIO DE TELEVISIÓN (mayo 2009): www.publico.es/publiscopio

RETIS, Jessica, LAMUEDRA, María y GARCÍA MATILLA, Agustín. (2019): Los informativos diarios en BBC y TV'. Madrid, Ediciones de la Torre.

TNS-Grupo Kantar (2009): Análisis de las preferencias entre las principales cadenas de televisión. Informe de resultados.

TNS-Grupo Kantar (2011): Preferencia en la elección de los canales de televisión. Imagen y posicionamiento de TVE.

TVE MARKETING (2011): Informe anual informativos. Ediciones/programas/tiempo 2011 VS. 2010

\section{Alicia GÓMEZ MONTANO}

Universidad Camilo José Cela (Madrid)

Profesora de Periodismo Televisivo

Periodista

amontano@ucjc.edu 